\title{
Evaluation of Infraspinatus Tendon Delamination Using Radial-Sequence Magnetic Resonance Imaging
}

\section{Hiroshi Negi ${ }^{1}$, Shin Yokoya ${ }^{2 *}$, Ryosuke Matsushita ${ }^{3}$, Norimasa Matsubara $^{4}$, Yuji Akiyama ${ }^{5}$ and Nobuo Adachi ${ }^{2}$}

${ }^{1}$ Department of Orthopaedic Surgery, Hiroshima-Nishi Medical Center, Hiroshima Japan

${ }^{2}$ Department of Orthopaedic Surgery, Graduate School of Biomedical and Health Sciences, Hiroshima University, Japan

${ }^{3}$ Department of Orthopaedic Surgery, Hiroshima Prefectural Hospital, Japan

${ }^{4}$ Department of Orthopaedic Surgery, Shobara Redcross Hospital, Japan

${ }^{5}$ Department of Clinical Radiology, Hiroshima University Hospital, Japan

*Corresponding Author: Shin Yokoya, Department of Orthopaedic Surgery,

Graduate School of Biomedical and Health Sciences, Hiroshima University, Japan.
Received: February 15, 2021

Published: February 26, 2021

(C) All rights are reserved by Shin Yokoya.,

et al.

\begin{abstract}
Objective: Magnetic resonance imaging (MRI) is used to diagnose rotator cuff tears; however, it is difficult to assess the delamination of the posterosuperior part of the insertion of the infraspinatus tendon on the axial, sagittal, and coronal views of conventional MRI. Therefore, in this study, we determined the accuracy of radial-sequence MRI for diagnosing the delamination of the posterosuperior part of the infraspinatus tendon.

Materials and Methods: In this retrospective study, the records of 126 rotator cuff tears in 126 patients (aged $65.5 \pm 9.7$ years; 79 males; 47 females) who underwent arthroscopic repair between June 2012 and March 2016 at our institution were considered. The intraoperative findings of the posterosuperior side of the infraspinatus tendon were compared with the preoperative conventional and radial-sequence MRI findings. The sensitivity and specificity of the two MRI methods were calculated, and their inter- and intraobserver reliability measured by independent orthopaedic surgeons were calculated using the kappa statistic $(\kappa)$.

Results: Intraoperatively, delamination of the infraspinatus tendon was noted in 32 shoulders. Conventional MRI had $40.1 \%$ sensitivity and 78.7\% specificity. Radial-sequence MRI had 75\% sensitivity and $94.7 \%$ specificity. The interobserver reliability and intraobserver reliability for radial-sequence MRI were both $\kappa=0.767$, corresponding to a high reproducibility, whereas for conventional MRI, these values were $\kappa=0.3$ and 0.588 , respectively, corresponding to a lower reproducibility.

Conclusion: Radial-sequence MRI shows higher accuracy and reproducibility for the diagnosis of delamination of the posterosuperior side of the infraspinatus tendon than conventional MRI. It is a useful tool to evaluate the delamination of the infraspinatus tendon.

Keywords: Radial-sequence Magnetic Resonance Imaging; Infraspinatus Tendon; Delamination; Rotator Cuff; Preoperative Diagnostic Imaging
\end{abstract}

\section{Abbreviations}

ABER: Abduction and External Rotation; DePPIT: Delamination of the Posterosuperior Part of the Infraspinatus Tendon; MRI: Magnetic Resonance Imaging; rMRI: Radial-Sequence MRI.

\section{Introduction}

Conventional magnetic resonance imaging (MRI) provides axial, coronal, and sagittal views of the examined body part. It is frequent- 
ly used to evaluate the rotator cuff, and delivers important information such as the size of cuff tears and the extent of fatty infiltration of the muscles. A comprehensive evaluation of the posterosuperior side of the infraspinatus tendon is nevertheless difficult using this method because the cleavage planes are not perpendicular to the rotator cuff insertion.

Delamination is observed as a horizontal tear between tendon layers, and is reported in many patients with rotator cuff tears [13] where it frequently occurs at the posterosuperior side of the rotator cuff. It is thought to be important to repair both delaminated layers to achieve optimal outcomes. As mentioned, the assessment of the posterosuperior side of the rotator cuff is limited on conventional MRI slices $[4,5]$.

The use of radial-sequence MRI (rMRI) was first reported by Kubo., et al. to diagnose acetabular labral tears in the hip joint [6]. Its effectivity was also reported in diagnosing tears of the glenoidal labrum and subscapularis tendon of the shoulder joint $[7,8]$. The rotator cuff tendons attach around the humeral head in a circular pattern; therefore, the perpendicular view of rMRI provides more information on the rotator cuff, including the posterosuperior part of the infraspinatus tendon. On the other hand, there no report focused on the effectiveness of the rMRI for diagnosing the delamination of infrasupinatus tendon.

In this study, it is hypothesised that rMRI provides more accurate information regarding delamination on the posterosuperior side of the infraspinatus tendon than conventional MRI. Therefore, the objective of this study was to investigate the accuracy of rMRI for diagnosing delamination of the posterosuperior part of the infraspinatus tendon (DePPIT).

\section{Material and Methods}

This diagnostic reliability study was reviewed and approved by the Ethical Committee (E-1448). Informed consent was obtained from all individual participants included in the study.

\section{Patients}

This retrospective study assessed 126 patients who underwent arthroscopic repair for rotator cuff tears along with preoperative MRI examinations between June 2012 and March 2016. Patients with previous shoulder surgery and those without preoperative MRIs were excluded. The mean age was 65.5 years (range, 34-84 years). Of the patients, 79 were male and 47 were female, and 84 right and 42 left shoulders were examined.

\section{Arthroscopic findings}

All arthroscopic examinations were performed by one orthopaedic surgeon with over 15 years of experience in shoulder surgery. The operation records and images were used to assess the presence of DePPIT retrospectively. A delamination tear was defined as a horizontal tear within the tendon resulting in two layers.

The intraoperative evaluation method was as follows: After the induction of general anaesthesia, patients were placed in the beach chair position. The posterior and anterior portals of the shoulder were established, and the glenohumeral joint was examined. If necessary, a debridement was performed before evaluating the cuff tear from the articular side. Consecutively, the bursal side was also examined and all arthroscopic findings through the posterior, posterolateral, and lateral viewing portals were documented. The presence of delamination was evaluated from the bursal side view and recorded in the surgical report (Figure 1).

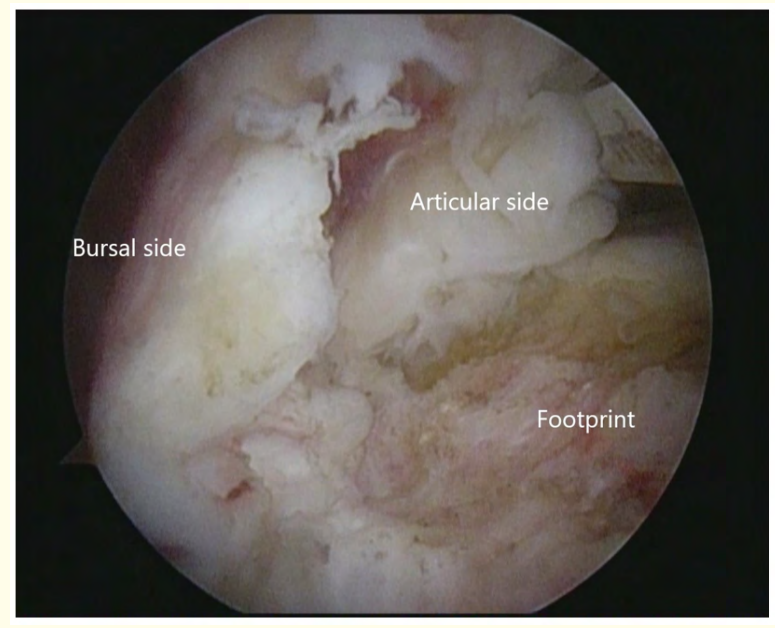

Figure 1: Representative arthroscopic view of an infraspinatus tendon with delamination from the bursal side view.

The bursal and the articular side of the delamination tear are marked. The footprint refers to the tendon insertion at the greater tuberosity.

\section{MRI protocol}

All MRIs were taken at the same time in a supine position with the shoulder slightly externally rotated using a shoulder coil. Four different MRI systems were used: Signa EXCITE HDxt 3.0T, GE 
Healthcare UK Ltd, Amersham Place, UK; Ingenia 1.5T MR system, Philips, North America LCC, Andover, MA, USA; Ingenia 3.0T MR system, Philips, North America LCC, Andover, MA, USA and Vantage Titan 3.0T, Canon Medical Systems Corporation, Tochigi, Japan. Both conventional and rMRI images were acquired in one session using the same MRI system for all images in an individual patient. The study included 47 patients examined with a $3.0 \mathrm{~T}$ MRI and 79 patients examined with a $1.5 \mathrm{~T}$ MRI.

The axial view was obtained using a T2-weighted sequence (reception time $=4,000 \mathrm{~ms}$; echo time $=100 \mathrm{~ms}$; echo train length $=22$; matrix $=304 \times 217$, slice thickness $=4.5 \mathrm{~mm}$ without gap; total scan duration $=2 \mathrm{~min}$ ). All continuous slices were taken perpendicular to the glenoid surface of the reference coronal images, which had been taken preliminarily. Oblique coronal images were also acquired using T2-weighted images (reception time $=4,000$ $\mathrm{ms}$; echo time $=100 \mathrm{~ms}$; echo train length $=22$; matrix $=320 \times 230$, slice thickness $=4.5 \mathrm{~mm}$ without gap, total scan duration $=2.24$ min). Continuous slices were taken perpendicular to the scapular spine on the axial view. The oblique sagittal images were acquired using T2-weighted images (reception time $=4,000 \mathrm{~ms}$; echo time $=$ $100 \mathrm{~ms}$; echo train length $=24$; matrix $=320 \times 230$, slice thickness $=4.5 \mathrm{~mm}$ without gap, total scan duration $=2.8 \mathrm{~min}$ ). The oblique slices were taken perpendicular to the oblique coronal images.

The rMRI images were acquired using T2-weighted images (reception time $=4,000 \mathrm{~ms}$; echo time $=100 \mathrm{~ms}$; echo train length $=$ 22 ; matrix $=352 \times 246$, slice thickness $=4.5 \mathrm{~mm}$ without gap; total scan duration $=2.56 \mathrm{~min}$ ). The centre of the humeral head was determined using the sagittal and coronal images. All slices were taken based on the sagittal images, perpendicular to the glenoid surface and passing through the centre of the humeral head with 24 slices at $7.5^{\circ}$ intervals (Figure 2 ).

\section{MRI evaluation of DePPIT}

The presence of DePPIT was evaluated using conventional MRI on T2-weighted images. On conventional MRI, DePPIT was defined as the presence of a high-intensity area in the infraspinatus tendon, whether there was continuity in the tendon insertion or not. The presence of DePPIT was examined by combining axial, coronal, and sagittal views and its presence was recorded (Figure 3).

Then, rMRI evaluation for DePPIT was performed. Posterosuperior slices beneath the scapular spine were used. When it was difficult to make a diagnosis using only one MRI slice, one or two additional slices adjacent to the posterosuperior slice were exam-

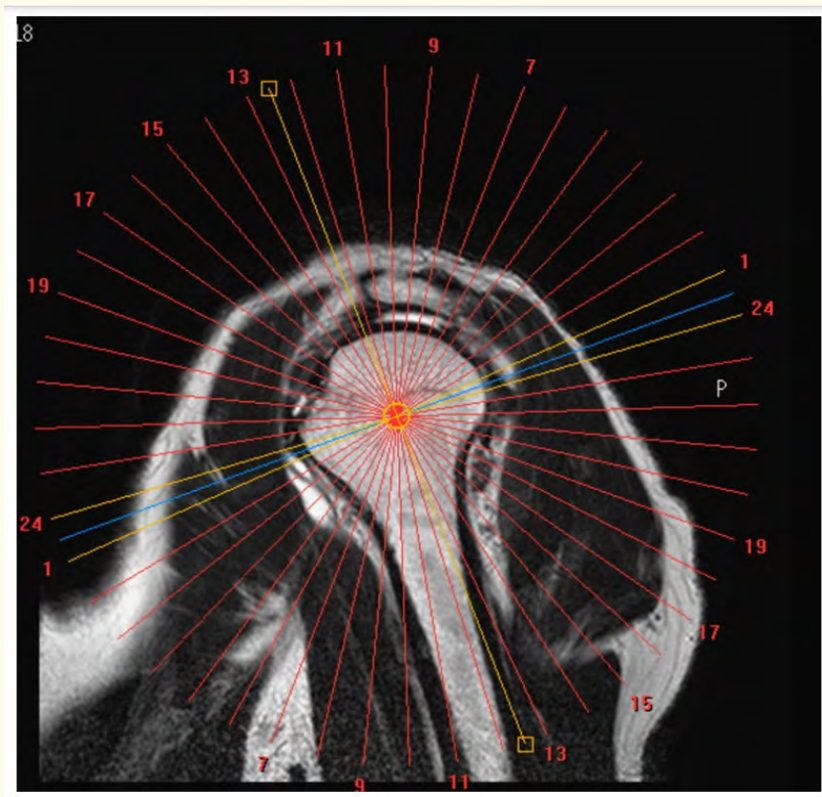

Figure 2: Illustration of the radial-sequence magnetic resonance imaging (MRI) planes used in 126 patients with rotator cuff tears.

All slices were taken based on the sagittal image, which was perpendicular to the glenoid surface passing through the centre of the humeral head with 24 slices at $7.5^{\circ}$ intervals.

ined. The insertion of the posterosuperior part of the infraspinatus tendon was classified into four grades according to the continuity of the tendon insertion and the presence of delamination as follows: Grade 0: intact cuff; Grade 1a: partial tear with delamination; Grade $1 \mathrm{~b}$ : partial tear without delamination; Grade 2a: complete tear with delamination and Grade $2 \mathrm{~b}$ : complete tear without delamination (Figure 4). Grade $1 \mathrm{a}$ and $2 \mathrm{a}$ were defined as the presence of DePPIT.

All images were evaluated by one orthopaedic surgeon with nine years of orthopaedic experience who was blinded to the patients and radiologist's reports, and who did not participate in the surgery. To reduce the influence of the evaluation with the conventional MRI, evaluation with the rMRI was performed at intervals of two weeks or more. We investigated the sensitivity, specificity, and accuracy of both the conventional MRI and rMRI findings by comparing them with the arthroscopic findings. 

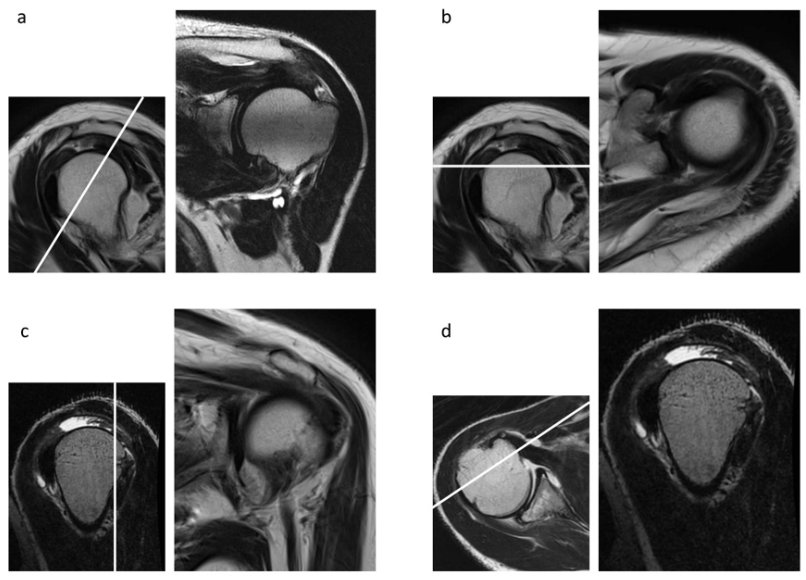

Figure 3: Illustration of the different magnetic resonance imaging (MRI) planes and views of the infraspinatus tendon used in 126 patients with rotator cuff tears.

(a) Radial-sequence MRI image with posterosuperior slices beneath the scapular spine; (b) Axial view of conventional MRI; (c) Coronal view of conventional MRI; (d) Sagittal view of conventional MRI. In the conventional MRI, (b), (c) and (d) were combined to evaluate the presence of delamination at the posterosuperior side of the infraspinatus tendon.

\section{Interobserver and intraobserver reliability}

All MR images were analysed blindly and independently by the same surgeon. To assess intraobserver reliability, he analysed all images twice with an interval of one month between the two assessments.

For evaluating interobserver reliability, images were evaluated by another orthopaedic surgeon with nine years of general orthopaedic experience.

\section{Statistical analysis}

Statistical analysis to assess the accuracy of combined MR images using axial, coronal, and oblique sagittal views in diagnosing DePPIT was performed by testing for equality of proportions between two samples. Significance was set at $\mathrm{P}<.05$. Interobserver and intraobserver reliability were analysed using the kappa statistic $(\kappa)$ defined as follows: $\kappa<0.4=$ poor; $0.4<\kappa<0.6=$ moderate; $0.6<\kappa<0.8=$ good; $0.75<\kappa=$ excellent [9]. All statistical analyses

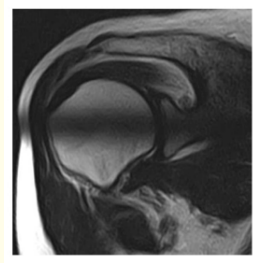

Grade 0

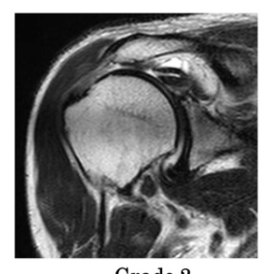

Grade 2

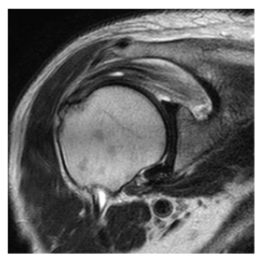

Grade 1a

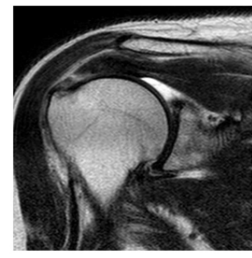

Grade 1b
Figure 4: Classification of the insertion of the posterosuperior part of the infraspinatus tendon.

Grade 0: Intact cuff; Grade 1a: Partial tear with

delamination; Grade 1b: Partial tear without delamination;

Grade 2a: Complete tear with delamination; Grade $2 \mathrm{~b}$ :

Complete tear without delamination. Grade $1 \mathrm{a}$ and 2 were

defined as presence of delamination of the posterosuperior part of the infraspinatus tendon. were performed using IBM SPSS Statistics for Windows, version 20 (IBM Corp., Armonk, NY, USA).

\section{Results}

During arthroscopic surgery, DePPIT was seen in 32 shoulders (25.4\%) and was absent in 94 shoulders (74.6\%). Conventional MRI showed DePPIT in 33 shoulders and no DePPIT in 93 shoulders. Among these, 20 findings were false positives, and 19 findings were false negatives when compared with the arthroscopy findings. The sensitivity of conventional MRI was $40.6 \%$, the specificity was $78.7 \%$, and accuracy was 69\% (Table 1).

rMRI showed 29 shoulders with DePPIT and 97 shoulders without DePPIT. The DePPIT classification based on rMRI classified 68 rotator cuffs (54\%) as Grade 0, 15 (11.9\%) as Grade 1a, 13 (10.3\%) as Grade $1 \mathrm{~b}, 14$ (11.1\%) as Grade 2a, and 16 (12.7\%) as Grade 2b. Five findings were false positives for DePPIT, and eight were false negatives when compared with the arthroscopy findings. The sen- 


\begin{tabular}{|l|c|c|}
\hline & \multicolumn{2}{|c|}{ MRI method } \\
\hline & rMRI(א) & Conventional MRI(к) \\
\hline Interobserver reliability & 0.767 & 0.3 \\
\hline Intraobserver reliability & 0.767 & 0.588 \\
\hline
\end{tabular}

Table 1: Interobserver and intraobserver reliability of MRI for evaluating the presence of delamination of the posterosuperior part of the infraspinatus tendon (DePPIT) in 126 patients undergoing arthroscopic rotator cuff repair.

Note: MRI: Magnetic Resonance Imaging; rMRI: Radial-sequence

MRI. sitivity was $75 \%$, specificity was $94.7 \%$, and accuracy was $89.7 \%$ (Table 1). The diagnostic sensitivity, specificity, and accuracy of rMRI were significantly higher than those of conventional MRI $(\mathrm{P}<$ $.05)$. The results for interobserver and intraobserver reliability are shown in table 2 . The interobserver and intraobserver reliability for rMRI were both $\kappa=0.767$ corresponding to high reproducibility and defined as excellent. The interobserver and intraobserver reliability of conventional MRI were $\kappa=0.3$ and 0.588 , respectively, corresponding to a lower reproducibility defined as poor and moderate, respectively.

\begin{tabular}{|l|c|c|c|c|c|c|c|c|}
\hline & & \multicolumn{2}{|c|}{ Arthroscopic findings } & Sensitivity & Specificity & Accuracy & PPV & NPV \\
\hline & & Delami (+) & Delami (-) & & & & & \\
\hline \multirow{2}{*}{ rMRI } & Delami (+) & 24 & 5 & 0.75 & 0.947 & 0.9 & 0.83 & 0.92 \\
\cline { 2 - 9 } & Delami (-) & 8 & 89 & & & & & \\
\hline \multirow{2}{*}{$\begin{array}{l}\text { Conventional } \\
\text { MRI }\end{array}$} & Delami (+) & 13 & 20 & 0.406 & 0.787 & 0.69 & 0.393 & 0.8 \\
\cline { 2 - 9 } & Delami (-) & 19 & 74 & & & & & \\
\hline
\end{tabular}

Table 2: MRI evaluation of the presence of DePPIT using rMRI and conventional MRI in 126 patients undergoing arthroscopic rotator cuff repair.

MRI: MAGNETIC RESONANCE IMAGING; RMRI: Radial-sequence MRI; Delami: Delamination; (+): present;

(-): absent; PPV: Positive predictive value; NPV: Negative predictive value.

\section{Discussion}

A delamination tear is thought to indicate degeneration of a tendon and is generally defined as a horizontal gap in the tendon [10]. Histologically, the horizontal split of the tendon occurs within layers, and necrotic changes are common at both the margins and apexes [11]. The horizontal tear of the tendon often shows endarteritis obliterans and a synovial-like lining. The synovial-like lining is thought to induce movement between the delaminated layers and hinder complete repair. It is therefore important to identify delamination in rotator cuff tears to remove the necrotic tissue prior to repair and ensure a conducive environment for healing between the layers.

In an anatomic study, Mochizuki., et al. reported that the infraspinatus tendon was involved in a large proportion of rotator cuff tears because the infraspinatus has a relatively wider footprint on the greater tuberosity than previously thought $[12,13]$. Cha., et al. and Gwak., et al. reported that both the superficial and deep layers were mainly retracted to the posteromedial side when involving the infraspinatus tendon $[14,15]$. Therefore, when managing delamination, it is important to evaluate the insertion of the posterosuperior side of the infraspinatus tendon.

In biomechanical studies, the superior capsule, which contains the capsular side of the infraspinatus tendon, played an important role in maintaining shoulder stability and, clinically, good results of superior capsular reconstruction were reported for irreparable rotator cuff tears [16-18]. Another biomechanical study revealed that a thicker superior capsular structure provides greater stability than a thinner one [19]. According to these reports, it is required to repair both layers of the delamination to create a thicker superior capsular structure.

Clinically, both Boileau., et al. and Flurin., et al. showed a negative relationship between the presence of delamination and a good clinical outcome [1,20]. Methods such as en masse repair and separate double-layer repair have been used in the repair of rotator cuff tears with delamination, and good clinical and functional results 
have been reported [21-23]. In many cases, repair of cuff tears is performed from the bursal side, where the bursal side layer is easier to see than the capsular side layer, which is also sometimes hidden because of medial retraction. A recent study showed the importance of the deeper layer for repair integrity [24]. Therefore, regardless of the repair technique, it is important to evaluate the presence of delamination and identify the capsular side layer during arthroscopic examination. If the presence or absence of delamination can be established even before surgery, this is very useful and can assist the preoperative planning for these patients.

Evaluating the posterosuperior side of the infraspinatus tendon is difficult on conventional MRI, because the slices are not perpendicular to the tendon insertion. Walz., et al. reported that only half of delamination tears have a visible cleavage plane in the interstitium on conventional MRI [5]. This study confirmed the difficulty in diagnosing DePPIT using conventional MRI. Choo., et al. reported the effectivity of MR arthrography in evaluating delaminated rotator cuff tears in the abduction and external rotation (ABER) position [25]. Although the technique showed high sensitivity and specificity, MR arthrography is invasive as it involves the injection of contrast agents with the possibility of allergic reactions to the agent. Moreover, maintaining the ABER position during MR arthrography may cause discomfort in some patients, especially those with rotator cuff tears, because the ABER position is close to the impingement position of the shoulder.

In the present study, rMRI was shown to be a non-invasive procedure with no risk of anaphylactic reactions. It had relatively higher accuracy than conventional MRI for evaluating delamination of the infraspinatus tendon. The additional examination time was acceptable (only 3-5 minutes per patient, depending on the experience of the radiological technician). Therefore, it can be concluded that $\mathrm{rMRI}$ is a valid and safe procedure to evaluate DePPIT.

The interobserver and intraobserver reliability of rMRI were classified as excellent in this study, whereas for conventional MRI, they were poor and moderate, respectively. This further underlines the difficulty of evaluating DePPIT using conventional MRI and the higher effectivity and accuracy of rMRI in diagnosing the condition. rMRI has also been reported to be an effective procedure for the evaluation of the subscapularis tendon and labrum tears [7]. Using rMRI, we can obtain comprehensive information about both delamination as well as other structural changes in the shoulder joint preoperatively.
There are some limitations to this study. This study was conducted retrospectively; prospective studies are needed to achieve higher levels of evidence. Furthermore, several models of either 1.5 $\mathrm{T}$ or 3.0 T MRI were used in this study. Nevertheless, the differences in accuracy between the conventional MRI and rMRI views were obvious with both magnet strengths. This suggests that even 1.5T MRI of each model was sufficient to investigate DePPIT with rMRI. Finally, rMRI resulted in some false-negative and false-positive cases. In the false-negative cases, the amount of synovial fluid that had collected between the laminated layers of the tendon was insufficient to be reflected on the rMRI images. In the false-positive cases, slices might have appeared as delaminated layers of the tendon where the infraspinatus showed fatty layers in the muscle because we used only T2-weighted images. Therefore, in clinical application, a large number of slices need to be evaluated to distinguish the delaminated layers of the tendon from fatty layers in the muscle on the T2-weighted image. Fat-suppression images may also be more effective in this regard.

\section{Conclusion}

In this study, radial-sequence MRI has shown a higher accuracy and reproducibility for the diagnosis of delamination of the posterosuperior side of the infraspinatus tendon than conventional MRI. Therefore, it can be considered a useful tool to evaluate the delamination of the infraspinatus tendon.

\section{Conflict of Interest}

The authors declare that they have no conflict of interest.

\section{Bibliography}

1. Boileau P., et al. "Arthroscopic repair of full-thickness tears of the supraspinatus: does the tendon really heal?". Journal of Bone and Joint Surgery 87.6 (2005): 1229-1240.

2. Flurin PH., et al. "Société Française d'Artroscopie. Réparation arthroscopique des ruptures transfixiantes de la coiffe des rotateurs: étude rétrospective multicentrique de 576 cas avec contrôle de la cicatrisation [Arthroscopic repair of fullthickness cuff tears: a multicentric retrospective study of 576 cases with anatomical assessment]". Revue de chirurgie orthopedique et reparatrice de l'appareil moteur 91 (2005): 31-42.

3. Sonnabend DH and Watson EM. "Structural factors affecting the outcome of rotator cuff repair". Journal of Shoulder and Elbow Surgery 11.3 (2002): 212-218. 
4. Han Y., et al. "Is posterior delamination in arthroscopic rotator cuff repair hidden to the posterior viewing portal?" Arthroscopy 29.11 (2013): 1740-1747.

5. Walz DM., et al. "MR imaging of delamination tears of the rotator cuff tendons". Skeletal Radiology 36.5 (2007): 411-416.

6. Kubo T., et al. "Radial-sequence magnetic resonance imaging in evaluation of acetabular labrum". Journal of Orthopaedic Science 4.5 (1999): 328-332.

7. Furukawa R., et al. "Diagnostic accuracy of magnetic resonance imaging for subscapularis tendon tears using radial-slice magnetic resonance images". Journal of Shoulder and Elbow Surgery 23.11 (2014): e283-290.

8. Honda H., et al. "Clinical application of radial magnetic resonance imaging for evaluation of rotator cuff tear". Orthopaedics and Traumatology: Surgery and Research 101.6 (2015): 715719.

9. Kundel HL and Polansky M. "Measurement of observer agreement”. Radiology 228.2 (2003): 303-308.

10. Hiroshige F. "The management of partial-thickness tears of the rotator cuff". Journal of Bone and Joint Surgery 85.1 (2003): 3-11.

11. Sonnabend DH., et al. "Laminated tears of the human rotator cuff: a histologic and immunochemical study". Journal of Shoulder and Elbow Surgery 10.2 (2001): 109-115.

12. Mochizuki T., et al. "Humeral insertion of the supraspinatus and infraspinatus. New anatomical findings regarding the footprint of the rotator cuff". Journal of Bone and Joint Surgery 90.5 (2008): 962-969.

13. Mochizuki T., et al. "Repair of rotator cuff tear with delamination: independent repairs of the infraspinatus and articular capsule". Arthroscopy Techniques 5.5 (2016): e1129-1134.

14. Cha SW., et al. "Retraction pattern of delaminated rotator cuff tears: dual-layer rotator cuff repair". Journal of Orthopaedic Surgery and Research 11.1 (2016): 75.

15. Gwak HC., et al. "Delaminated rotator cuff tear: extension of delamination and cuff integrity after arthroscopic rotator cuff repair". Journal of Shoulder and Elbow Surgery 24.5 (2015): 719-726.

16. Ishihara Y., et al. "Role of the superior shoulder capsule in passive stability of the glenohumeral joint". Journal of Shoulder and Elbow Surgery 23.5 (2014): 642-648.

17. Mihata T., et al. "Clinical results of arthroscopic superior capsule reconstruction for irreparable rotator cuff tears". Arthros- copy 29.3 (2013): 459-470.

18. Mihata T., et al. "Superior capsule reconstruction to restore superior stability in irreparable rotator cuff tears: a biomechanical cadaveric study". American Journal of Sports Medicine 40.10 (2012): 2248-2255.

19. Mihata T., et al. "Biomechanical effect of thickness and tension of fascia lata graft on glenohumeral stability for superior capsule reconstruction in irreparable supraspinatus tears". Arthroscopy 32.3 (2016): 418-426.

20. Flurin P-H., et al. "Cuff integrity after arthroscopic rotator cuff repair: correlation with clinical results in 576 cases". Arthroscopy 23.4 (2007): 340-346.

21. Sugaya H., et al. "Repair integrity and functional outcome after arthroscopic double-row rotator cuff repair. A prospective outcome study". Journal of Bone and Joint Surgery 89.5 (2007): 953-960.

22. Hepp P., et al. "Knotless anatomic double-layer double-row rotator cuff repair: a novel technique re-establishing footprint and shape of full-thickness tears". Archives of Orthopaedic and Trauma Surgery 129.8 (2009): 1031-1036.

23. Park JY., et al. "Clinical and ultrasonographic outcomes of arthroscopic suture bridge repair for massive rotator cuff tear". Arthroscopy 29.2 (2013): 280-289.

24. Kim YK., et al. "Morphologic factors related to repair outcomes for delaminated rotator cuff tears: a minimum 2-year retrospective comparison study". Arthroscopy 35.2 (2019): 332340.

25. Choo HJ., et al. "Delaminated tears of the rotator cuff: prevalence, characteristics, and diagnostic accuracy using indirect MR arthrography". AJR American Journal of Roentgenology 204.2 (2015): 360-366.

\section{Assets from publication with us}

- Prompt Acknowledgement after receiving the article

- Thorough Double blinded peer review

- Rapid Publication

- Issue of Publication Certificate

- High visibility of your Published work

Website: $\underline{w w w . a c t a s c i e n t i f i c . c o m / ~}$

Submit Article: www.actascientific.com/submission.php Email us: editor@actascientific.com

Contact us: +919182824667 